\title{
Gentamicin Pharmacokinetics in the Chicken Inner Ear
}

\author{
Eric C. Bunting, ${ }^{1}$ Debra L. Park, ${ }^{2}$ Dianne Durham, ${ }^{2}$ and Douglas A. Girod ${ }^{2}$ \\ ${ }^{1}$ Kansas Ear Nose Throat, Wichita, KS 67211, USA \\ ${ }^{2}$ University of Kansas Medical Center, Department of Otolaryngology-Head and Neck Surgery, Kansas City, KS 66160, USA
}

Received: 21 August 2003; Accepted: 10 November 2003; Online publication: 4 March 2004

\section{ABSTRACT}

Avians have the unique ability to regenerate cochlear hair cells that are lost due to ototoxins or excessive noise. Many methodological techniques are available to damage the hair cells for subsequent scientific study. A recent method utilizes topical application of an ototoxic drug to the round window membrane. The current study examines the pharmacokinetics of gentamicin in the inner ear of chickens following topical application to the round window membrane or a single systemic high dose given intraperitoneally. Chickens were given gentamicin topically or systemically and survived for $1,4,12,24$, or $120 \mathrm{~h}$ (controls at 4 and $120 \mathrm{~h}$ ). Serum and perilymph samples were obtained prior to sacrifice and measured for gentamicin levels. Results revealed higher levels of gentamicin in the perilymph of topically treated chickens than systemically treated chickens, with significant amounts of gentamicin still present in both at the latest survival time of 5 days. As expected, systemically treated chickens had much higher levels of gentamicin in the serum than topically treated chickens. Advantages and disadvantages to each method of drug administration are discussed.

Keywords: pharmacokinetics, gentamicin, avian, topical, systemic

Correspondence to: Debra L. Park - University of Kansas Medical Center - Department of Otolaryngology-Head and Neck Surgery • Mailstop \#3010 • 3901 Rainbow Blvd. • Kansas City, KS 66160 • Telephone: (913) 588-6714; Fax: (913) 588-6708; email: dpark@, kumc.edu

\section{INTRODUCTION}

Unlike the human cochlea, the sensory epithelium of the chicken cochlea (basilar papilla) has the ability to regenerate hair cells after damage from an ototoxic drug (Cotanche et al. 1994; Smolders 1999; Tsue et al. 1994). This regeneration leads to anatomical and progressive physiologic recovery (Girod et al. 1991; Marean et al. 1993; Tucci et al. 1990). In birds, many studies have shown the effects of the ototoxic drug gentamicin with the resulting hair cell regeneration, but the initiating mechanism or sequence leading to recovery has yet to be determined (Cotanche et al. 1994; Tsue et al. 1994). Once determined, the hope would be to find a mechanism that can be stimulated or activated in the human population.

The systemic injection of gentamicin has been a common research tool to create cochlear hair cell damage in the chicken. It has been noted that when given systemically, gentamicin produces variable cochlear damage and its systemic effects may also produce damage in the central auditory system (Girod et al. 1991; Lippe et al. 1991). The amount of damage that can be created by a systemic injection is limited to the basal $40 \%-50 \%$ of the chicken cochlea due to the nephrotoxicity of gentamicin at higher doses. Another method to induce cochlear hair cell damage involves the technique of applying gentamicin directly to the round window to increase the amount of damage to the cochlea while limiting the systemic and nephrotoxic effects (Husmann et al. 1998).

Husmann et al. (1998) studied several variables in the placement and dosage of gentamicin at the round window. The placement of gentamicin at the round window membrane in a solid matrix was necessary to produce consistent damage to the cochlear hair cells. 
The damage to the hair cells seemed to be more dose dependent rather than time dependent with exposures greater than $30 \mathrm{~min}$. The damage to the cochlear hair cells via the round window technique was found to be much greater than the damage that could be produced with a systemic injection of gentamicin. A $1 \mathrm{mg}$ dose of gentamicin placed at the round window reliably created $75 \%-100 \%$ damage to the cochlear hair cells, while smaller doses had less damage and increased variability (Husmann et al. 1998). However, the pharmacokinetics of gentamicin were not evaluated in our 1998 study. The current study was designed to evaluate the pharmacokinetics of gentamicin in the avian inner ear with both the round window application and systemic injection.

\section{METHODS}

Broiler chicks of the Avian $\times$ Peterson strain ( $n=116)$ were the subjects of this experiment. Birds were obtained as day-old hatchlings from a commercial supplier (ConAgra, Batesville, AR) and were subsequently housed in an AAALAC-approved facility with ad libitum access to food and water. All experimental protocols were approved by the KUMC Institutional Animal Care and Use Committee (IACUC).

The birds were separated into groups by method of drug administration. One group received a systemic intraperitoneal injection of gentamicin $(200 \mathrm{mg} / \mathrm{kg})$ or saline (equivalent volume). The other group received gentamicin or saline topically applied to the round window via Gelfoam pledget. All birds were between the ages of 11 and 21 days at the time of treatment and were fasted from food the night prior to drug administration.

The gentamicin solution was prepared using gentamicin sulfate, distilled water, sodium bisulfate and EDTA (all chemicals from Sigma, St. Louis, MO). The concentrations of gentamicin differed for the two test groups. The systemic gentamicin was prepared to a concentration of $40 \mathrm{mg} / \mathrm{ml}$ and the birds were dosed according to weight $(200 \mathrm{mg} / \mathrm{kg})$. This dosage of gentamicin creates maximal cochlear hair cell damage without significant mortality from the associated nephrotoxicity. The gentamicin solution used in the round window technique allowed the administration of $1 \mathrm{mg}$ of gentamicin ( $5 \mu \mathrm{l} \mathrm{of} \mathrm{a} 200 \mathrm{mg} / \mathrm{ml}$ solution) to a small Gelfoam pledget (Upjohn Company, Kalamazoo, MI). All birds were dosed similarly in the round window group with no variation for weight, with each receiving a $1 \mathrm{mg}$ dose to the round window membrane.

\section{Systemic administration of gentamicin or saline}

The systemic group had gentamicin sulfate (200 mg/ $\mathrm{kg}$ ) or saline (equivalent volume) injected intraperitoneally to the right lower quadrant of the abdomen. Perilymph was obtained from chicks $1,4,12,24$, or $120 \mathrm{~h}$ after gentamicin injection, and 4 or $120 \mathrm{~h}$ after saline injection. The number of chicks per group was $8,13,9,9$, and 8 and 5 and 5 , respectively. Under anesthesia, the tympanic membrane was visualized through the ear canal and a small myringotomy was then created in the tympanic membrane just anterior to the columella. The columella (middle ear ossicle) was isolated and removed from the oval window using microsurgical forceps. This allowed access to the adjacent round window. Perilymph $(5 \mu \mathrm{l})$ was harvested through the round window using a microliter syringe. The same technique was used in the contralateral ear to obtain perilymph for comparison. The birds, while under anesthesia, then had intracardiac blood obtained prior to sacrifice for serum measurements. All samples were then processed and analyzed for gentamicin levels.

\section{Topical administration of gentamicin or saline}

The round window topical administration technique utilized a Gelfoam pledget as a solid matrix to deliver gentamicin or saline to the round window. All birds in this group received $1 \mathrm{mg}$ of gentamicin solution (controls received equivalent volumes of saline) that was soaked up by a Gelfoam pledget weighing $0.4 \mathrm{mg}$. Birds were anesthetized with ketamine $(35 \mathrm{mg} / \mathrm{kg})$ and nembutal $(19 \mathrm{mg} / \mathrm{kg})$. After anesthesia, the round window of the right ear was exposed by a small myringotomy anterior to the columella. The pledget was placed at the round window and its location confirmed by direct visualization. The pledget was left in place for $2 \mathrm{~h}$ and then removed with microsurgical forceps. Birds were placed in cages with heat lamps during this recovery time and most were awake within an hour. For birds that were awake, an additional $1 / 4$ to $1 / 2$ dose of ketamine and nembutal was given prior to removing the pledget. The perilymph was sampled at $1,4,12,24$, or $120 \mathrm{~h}$ after gentamicin pledget placement from both the right and the left ear, the left ear serving as an intrabird control. Bilateral samples were taken 4 or $120 \mathrm{~h}$ after saline pledget placement. The number of chicks per group was 10,9, 9, 8, and 13 and 5 and 5, respectively. No perilymph was sampled from the chickens with topically administered gentamicin or saline at the $1 \mathrm{~h}$ time point because the pledget was not removed until $2 \mathrm{~h}$ after placement. Intracardiac blood samples were obtained prior to sacrifice for serum analysis. The perilymph and serum samples 
were then processed and gentamicin levels were measured.

\section{Perilymph acquisition and gentamicin assay}

Perilymph $(5 \mu \mathrm{l})$ was harvested through the round window using a microliter syringe and 30 gauge needle (Hamilton Co., Reno, NV). The needles were disposed of after one use. The syringes were cleaned by pulling $100 \%$ ethanol through the syringe two times, followed by pulling double-distilled water through the syringe three times. Prior to collecting samples for this experiment, the cleaning procedure was tested. After drawing perilymph from a chicken that was topically treated with gentamicin, the cleaning procedure was utilized. Then the same needle and syringe was used to draw $5 \mu \mathrm{l}$ of double-distilled water, which was tested for gentamicin levels using the fluorescence polarization immunoassay. Results revealed no measurable gentamicin. Thus, we determined this cleaning procedure was adequate for the experiment.

After removal of the gentamicin pledget, the round window membrane was not flushed with saline. The first perilymph sample was taken $2 \mathrm{~h}$ after pledget removal. Thus, it is possible that there was residual gentamicin remaining on the round window membrane that was subsequently collected as the needle passed through the membrane to collect the perilymph. This was likely a trace amount, since a 30 gauge needle was used and has a very small perimeter. Unfortunately, no controls were employed to test this possibility, so it is possible that the absolute amounts of gentamicin we report are slightly higher than those truly present in the perilymph.

Perilymph and serum samples were sent to our hospital's in-patient laboratory for gentamicin testing. The University of Kansas Medical Center laboratory processed and analyzed the samples for gentamicin levels using a fluorescence polarization immunoassay, TDx FLx, from Abbott Laboratories (Abbott Park, IL). This is the same assay used for clinical purposes.

\section{Preparation of cochlear tissue}

All cochleae (including those from saline-treated control animals) were prepared at the time of death for scanning electron microscopy (SEM) to assess cochlear damage as previously described (Girod et al. 1989). Sacrifice was achieved using an intracardiac dose of Euthasol. Each ear was perfused with 3.5\% glutaraldehyde in phosphate-buffered saline. The head was then placed in the same glutaraldhyde fix at $4^{\circ} \mathrm{C}$ for at least 5 days. The temporal bone containing the cochlea then was dissected free from the head

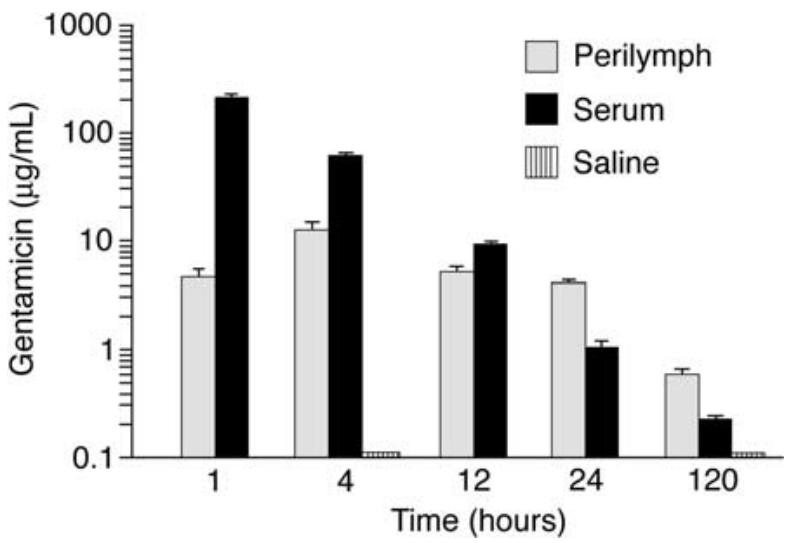

FIG 1. Gentamicin concentrations in the gentamicin-treated chickens' perilymph and serum and the saline-treated chickens' perilymph following a systemic intraperitoneal injection. The measurements were completed at increasing time periods after injection (1, 4, 12, 24, and $120 \mathrm{~h}$ ). A logarithmic scale is employed to display the large differences between levels in the serum and perilymph. At $120 \mathrm{~h}$, more gentamicin continues to be measured in the perilymph than the serum or controls. Error bars represent standard error of the mean.

and postfixed with $1 \%$ osmium in distilled water. The final dissection included removing the tectorial membrane. Preparation for the scanning electron microscope included dehydration with ethanol, critical point drying in carbon dioxide, and sputtercoating with palladium gold. Scanning electron micrographs of the cochlear surface were taken at a magnification of $110 \times$. Montages were then assembled to assess the entire cochlear surface for damage.

Gentamicin-induced cochlear hair cell damage always occurs in a basal to apical progression. The percent length damage was calculated from the measurement of the damaged cochlear length relative to total length of the cochlea via scanning electron micrograph montages (Husmann et al. 1998).

\section{RESULTS}

\section{Systemic administration of gentamicin or saline}

The birds in the systemic gentamicin group all survived intraperitoneal injection without significant complication or mortality. The gentamicin levels detected in the serum and perilymph are shown in Figure 1. Note that the $y$ axis is on a logarithmic scale. This allows for easier visualization of the differences between groups when the range of data is large. The serum shows rapid uptake of gentamicin with the mean peak value at $1 \mathrm{~h}$ post-injection measuring $216 \mu \mathrm{g} / \mathrm{ml}$. The mean perilymph concentration (bilateral average) shows a delay relative to serum in reaching the peak value of $13 \mu \mathrm{g} / \mathrm{ml}$ at $4 \mathrm{~h}$ post-injection. The kidneys rapidly clear the serum of gen- 
tamicin, as serum levels have dropped to a concentration of $9 \mu \mathrm{g} / \mathrm{ml}$ at the $12 \mathrm{~h}$ post-injection time period. The perilymph is delayed in its clearance of gentamicin and actually surpasses serum levels at $24 \mathrm{~h}$ post-injection. Both the serum $(0.23 \mu \mathrm{g} / \mathrm{ml})$ and perilymph $(0.59 \mu \mathrm{g} / \mathrm{ml})$ show very low levels at the $120 \mathrm{~h}$ post-injection time period.

Control birds that received systemic injections of saline showed very low levels of gentamicin in the serum $(0.22$ and $0.20 \mu \mathrm{g} / \mathrm{ml})$ and perilymph $(0.10$ and $0.07 \mu \mathrm{g} / \mathrm{ml}$-average of right and left ears) at both the 4 and $120 \mathrm{~h}$ time periods, respectively.

Statistics on systemic data. To examine the data statistically, a one-way ANOVA was performed on the gentamicin levels in the perilymph across time. It showed significant differences between times $(p<$ $0.0001)$. Fisher's post hoc tests revealed that gentamicin levels are significantly higher after $4 \mathrm{~h}$ compared with $1 \mathrm{~h}(p=0.0002), 12 \mathrm{~h}(p=0.0002), 24 \mathrm{~h}$ $(p<0.0001)$, and $120 \mathrm{~h}(p<0.0001)$. Fisher's post hoc testing also showed more gentamicin at $12 \mathrm{~h}$ compared with that at $120 \mathrm{~h}(p=0.0340)$. These results confirm that the peak levels of gentamicin in the perilymph are reached $4 \mathrm{~h}$ after systemic injection.

A one-way ANOVA was also performed on the gentamicin levels in the serum from gentamicintreated chickens across time. It too showed significant differences between times $(p<0.0001)$. Fisher's post hoc tests revealed that gentamicin levels are significantly higher at $1 \mathrm{~h}$ compared with $4,12,24$, and $120 \mathrm{~h}$ (all $p<0.0001$ ). Fisher's post hoc tests also revealed higher levels of gentamicin after $4 \mathrm{~h}$ compared with 12, 24, and $120 \mathrm{~h}$ (all $p<0.0001$ ). These results confirm that the peak levels of gentamicin in the serum are reached $1 \mathrm{~h}$ after systemic injection.

A second set of analyses was performed on the data shown in Figure 1 to examine the levels of gentamicin between the locations sampled (perilymph, serum, perilymph from saline-treated) at each survival time. $T$ tests were performed between perilymph and serum at 1, 12, and $24 \mathrm{~h}$. ANOVAs with Fisher's post hoc tests were performed between perilymph, serum, and saline at 4 and $120 \mathrm{~h}$. At $1 \mathrm{~h}$, gentamicin is significantly higher in the serum than perilymph $(p<$ $0.0001)$. At 4 h Fisher's post hoc tests show significantly more gentamicin in serum than in perilymph $(p<$ $0.0001)$ and saline $(p<0.0001)$. At $12 \mathrm{~h}$, gentamicin is significantly higher in serum than in perilymph $(p<$ $0.0038)$. At $24 \mathrm{~h}$, gentamicin levels in the serum drop significantly below those in the perilymph $(p<$ $0.0001)$. At $120 \mathrm{~h}$, Fisher's post hoc tests show gentamicin levels in the perilymph continue to significantly exceed those in the serum $(p=0.0002)$ and saline $(p<0.0001)$.

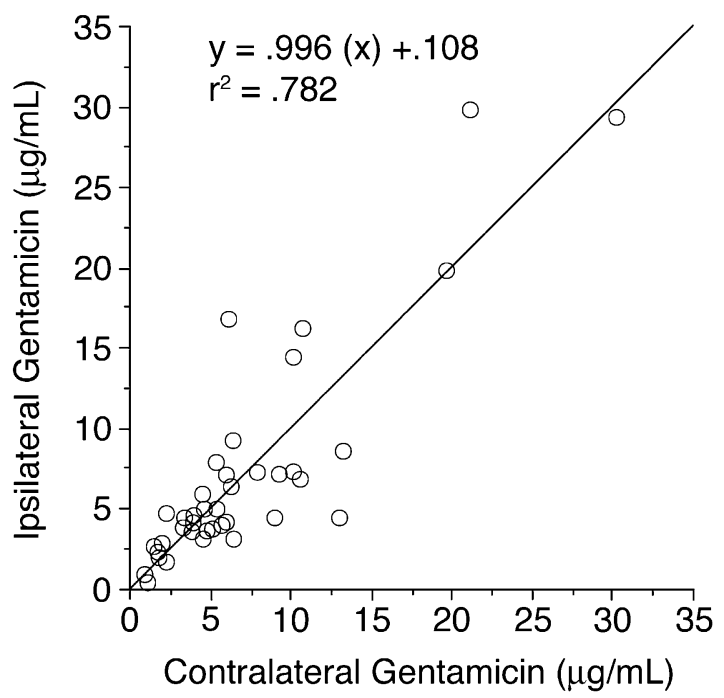

FIG. 2. Linear regression analysis shows a significant relationship in drug levels seen between gentamicin-treated chickens' right and left inner ears $\left(p<0.001 ; r^{2}=0.782\right)$ following a single systemic dose.

In the group treated with systemic gentamicin, the perilymph values from the right and left cochleae were both measured to correlate intrabird variability. Linear regression analysis between the gentamicin levels in the right and left perilymph show a statistically significant relationship $(p<0.001)$ with an $r^{2}$ of 0.782 (Fig. 2). This relationship has also been noted anatomically with similar patterns of damage seen in both cochleae of the same bird following systemic administration of gentamicin.

Anatomy from systemically treated chickens. The SEM montages show normal-appearing cochleae at the $24 \mathrm{~h}$ post-injection time period (Fig. 3a). The cochlea shows ordered stereocilia with no evidence of damage. At $120 \mathrm{~h}$ after gentamicin injection, the basal $40 \%$ of the chicken cochlea has been damaged and the sensory epithelium is absent of mature hair cells, except for a few extruding mature hair cells (Fig. 3b). The SEM montages that were evaluated in our saline-controlled systemic injection group were anatomically normal.

\section{Topical administration of gentamicin or saline}

Birds undergoing gentamicin pledget placement at the round window had no significant complication relating to their myringotomy and $2 \mathrm{~h}$ gentamicin pledget exposure. At the $1 \mathrm{~h}$ gentamicin exposure period, samples were obtained only from the contralateral cochlear perilymph and serum. The ipsilateral cochlear perilymph was not evaluated at the $1 \mathrm{~h}$ period to avoid obtaining spuriously elevated 

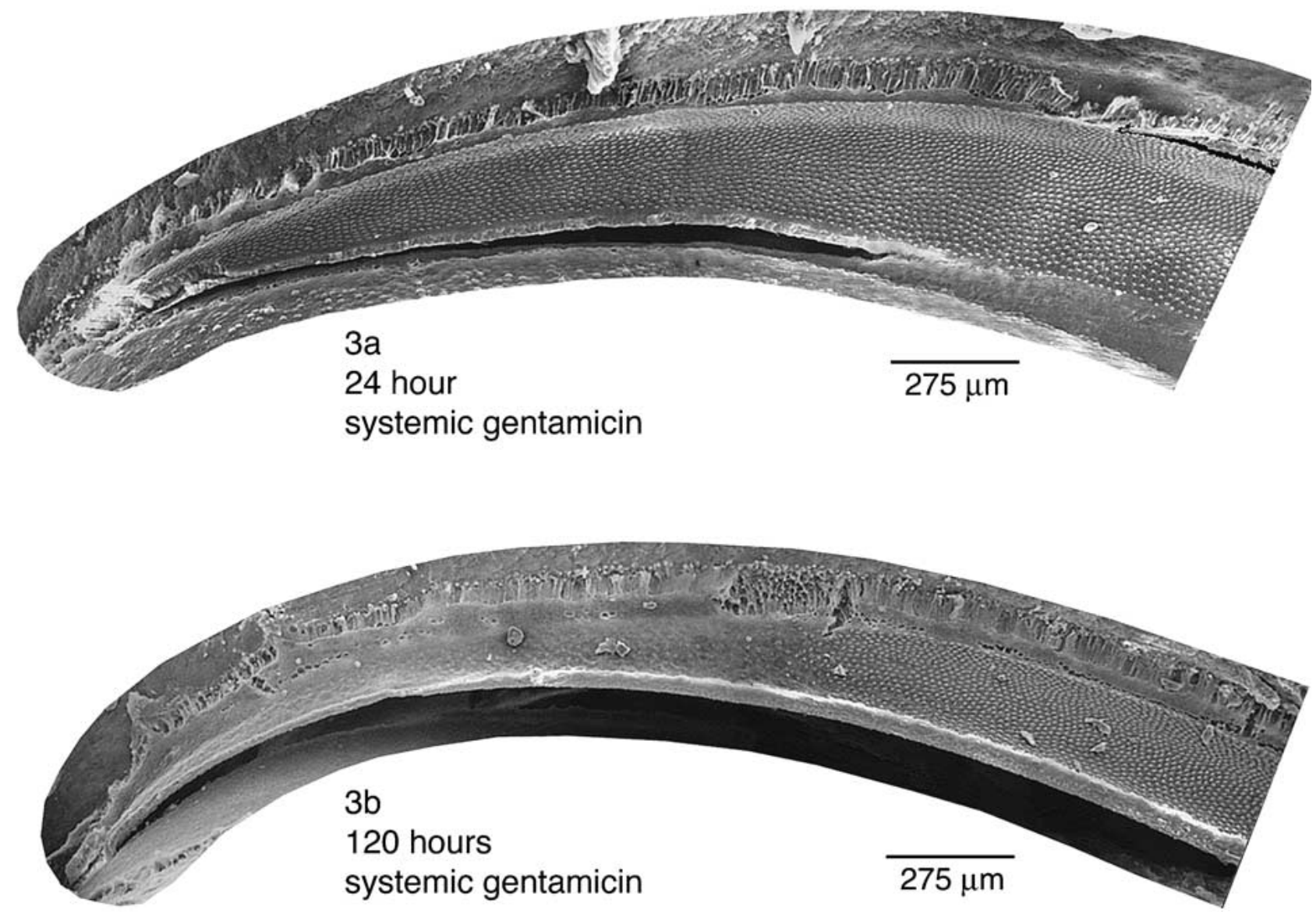

FIG. 3. Montages of SEM images from cochleae (figure shows approximately the basal half of the cochlea) following systemic injections of gentamicin $(200 \mathrm{mg} / \mathrm{kg})$ at (a) $24 \mathrm{~h}$ and (b) $120 \mathrm{~h}$ following intraperitoneal injection. The hair cells in (a) are of normal appear-

gentamicin levels because the pledget was still in place. Both the contralateral cochlear perilymph and serum had very low levels of gentamicin present at this early time period (Fig. 4; also with a logarithmic scale on the $y$ axis). At the $4 \mathrm{~h}$ gentamicin exposure time period ( $2 \mathrm{~h}$ after removal of pledget), the ipsilateral cochlear perilymph had a mean peak gentamicin concentration of $55 \mu \mathrm{g} / \mathrm{ml}$. The contralateral cochlear perilymph and serum gentamicin levels remained at low but detectable levels. The contralateral cochlear perilymph exceeds serum gentamicin levels by $4 \mathrm{~h}$ and persists throughout the time periods tested. The perilymph gentamicin concentration in the ipsilateral cochlea was still elevated to high levels $24 \mathrm{~h}$ after exposure. The perilymph had cleared the gentamicin by the $120 \mathrm{~h}$ post-exposure time period to low but still detectable levels.

Chickens that were treated with saline-soaked pledgets placed at the round window had no appreciable gentamicin in the serum, saline-treated ipsilateral cochlear perilymph, or the untreated ance without signs of damage. The mature hair cells in (b) have been damaged and subsequently lost in the basal 40 of the cochleae with only a few blebs present that demonstrate extruded hair cells.

contralateral cochlear perilymph (4 h: $0.29,0,0.26$; $120 \mathrm{~h}: 0.25,0.27,0.22$; respectively, in $\mu \mathrm{g} / \mathrm{ml}$ ).

Statistics on topical data. Statistical analyses of the data from the topically treated chickens (Fig. 4) are similar to those from the systemically treated chickens (Fig. 1). A one-way ANOVA was performed on the gentamicin levels in the ipsilateral perilymph across time. It showed significant differences between times $(p<0.0001)$. Fisher's post hoc tests revealed that gentamicin levels are significantly higher after 4 and $12 \mathrm{~h}$ compared with $24 \mathrm{~h}$ $(p=0.0150$ and 0.0488$)$ and $120 \mathrm{~h}(p<0.0001$ and $0.0001)$. Fisher's post hoc testing also showed significantly more gentamicin at $24 \mathrm{~h}$ than at $120 \mathrm{~h}$ $(p=0.0407)$.

Another one-way ANOVA was performed on the gentamicin levels in the contralateral perilymph across time. It showed significant differences between times $(p<0.0001)$. Fisher's post hoc tests revealed that gentamicin levels are significantly lower at $1 \mathrm{~h} \mathrm{com-}$ pared with 4,12 , and $24 \mathrm{~h}(p=0.0002,0.0003$, and $0.0011)$. The levels then drop back to significantly 


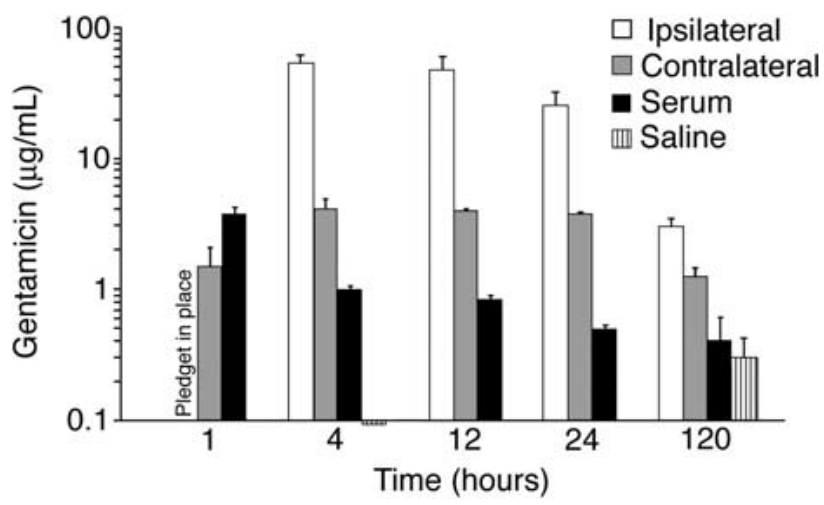

FIG. 4. Gentamicin concentrations in the gentamicin-treated chickens' ipsilateral perilymph, contralateral perilymph, and serum and the saline-treated chickens' perilymph following topical drug administration. The measurements were completed at increasing time periods after injection $(1,4,12,24$, and $120 \mathrm{~h})$. At the $1 \mathrm{~h}$ time period, a measurement from the ipsilateral perilymph was not obtained because the pledget was still in place. A logarithmic scale is employed to display the large differences between levels in the ipsilateral perilymph and the other groups. At $120 \mathrm{~h}$, more gentamicin continues to be measured in the ipsilateral perilymph than the other groups. Error bars represent standard error of the mean.

lower levels at $120 \mathrm{~h}$ compared with 4,12 , and $24 \mathrm{~h}$ $(p<0.0001, p=0.0002, p>0.0001)$.

A final one-way ANOVA was performed on the gentamicin levels in the serum from gentamicintreated chickens across time. It showed significant differences between times $(p<0.0001)$. Fisher's post $h o c$ tests revealed significantly increased gentamicin at $1 \mathrm{~h}$ compared with $4,12,24$, and $120 \mathrm{~h}$ (all $p<$ $0.0001)$.

A second set of analyses was performed on the data shown in Figure 4 to examine the levels of gentamicin between the locations sampled (ipsilateral perilymph, contralateral perilymph, serum from gentamicintreated, perilymph from saline-treated) at each survival time (5 one-way ANOVAs with Fisher's post hoc tests). At $1 \mathrm{~h}$, no data are available from the ipsilateral group because the pledget is still in place at that time. A $t$ test showed significantly more gentamicin in the serum than the contralateral perilymph $(p=0.0075)$. At $4 \mathrm{~h}$, post hoc tests showed significantly more gentamicin in the ipsilateral measurements than the contralateral, serum, and saline measurements (all $p<$ $0.0001)$. At $12 \mathrm{~h}$, significantly more gentamicin is in the ipsilateral measurements than the contralateral or serum measurements $(p=0.0002$ and $p<0.0001)$. Similarly at $24 \mathrm{~h}$, significantly more gentamicin is in the ipsilateral measurements than the contralateral or serum measurements $(p=0.0013$, and 0.0003$)$. Finally at $120 \mathrm{~h}$, the ipsilateral measurements continue to have gentamicin levels significantly exceeding contralateral, serum, and saline measurements $(p=0.0002, p<0.0001, p<0.0001)$.
Anatomy from topically treated chickens. No damage was seen in SEM montages from chickens in this study killed 4 or $12 \mathrm{~h}$ after topical gentamicin treatment. In previous studies, we have observed some hair cell damage at a $12 \mathrm{~h}$ survival period for topically treated chickens (enlarged hair cell surface area, disoriented hexagonal arrangement, some extruded hair cells) but never at a $4 \mathrm{~h}$ survival period. We have not examined the cochleae at the transmission electron microscopic level, which may show other changes not seen in scanning electron micrographs.

The SEM montage from the $24 \mathrm{~h}$ round window gentamicin exposure shows a significant amount of damage to the sensory epithelium (Fig. 5a). Approximately $75 \%$ (average of all cochleae) of the distance from the basal end and extending toward the apical end of the cochlea shows evidence of hair cell damage. The cochleae examined at the $120 \mathrm{~h}$ time period show evidence of near-total ablation of mature hair cells (Fig. 5b). SEM montages (not shown) were carefully examined from the contralateral cochleae. We found no evidence of damaged hair cells in any of the control cochleae.

\section{Topical vs. systemic administration of gentamicin}

An equality of variance $F$ test was completed to determine if the two methods (round window application vs. systemic injection of gentamicin) produced different degrees of variability in perilymph levels. The round window gentamicin application has significantly more variability in the perilymph gentamicin levels than the systemic administration perilymph gentamicin levels $(p<0.001)$.

Since our assay detected gentamicin in the serum and perilymph of chickens treated with saline (topical or systemic), we tested for differences between these groups. At $4 \mathrm{~h}$, no statistically significant differences were found in the amount of gentamicin in the ipsilateral perilymph between systemic and topical saline treatments ( $t$ test, $p=0.142$ ), or in the contralateral perilymph between systemic and topical saline treatments ( $t$ test, $p=0.308$ ), or in the serum between systemic and topical saline treatments ( $t$ test, $p=0.144)$. There were also no statistically significant differences for these same comparisons at $120 \mathrm{~h}$ $(p=0.241, p=0.187, p=0.389)$.

\section{DISCUSSION}

Although gentamicin is a well known and often studied ototoxic agent, the pharmacokinetics of this drug in the inner ear are not well understood. Treating the inner ear directly with ototoxic drugs has regained the attention of clinical otologists over the past several years as a treatment tool for vestib- 

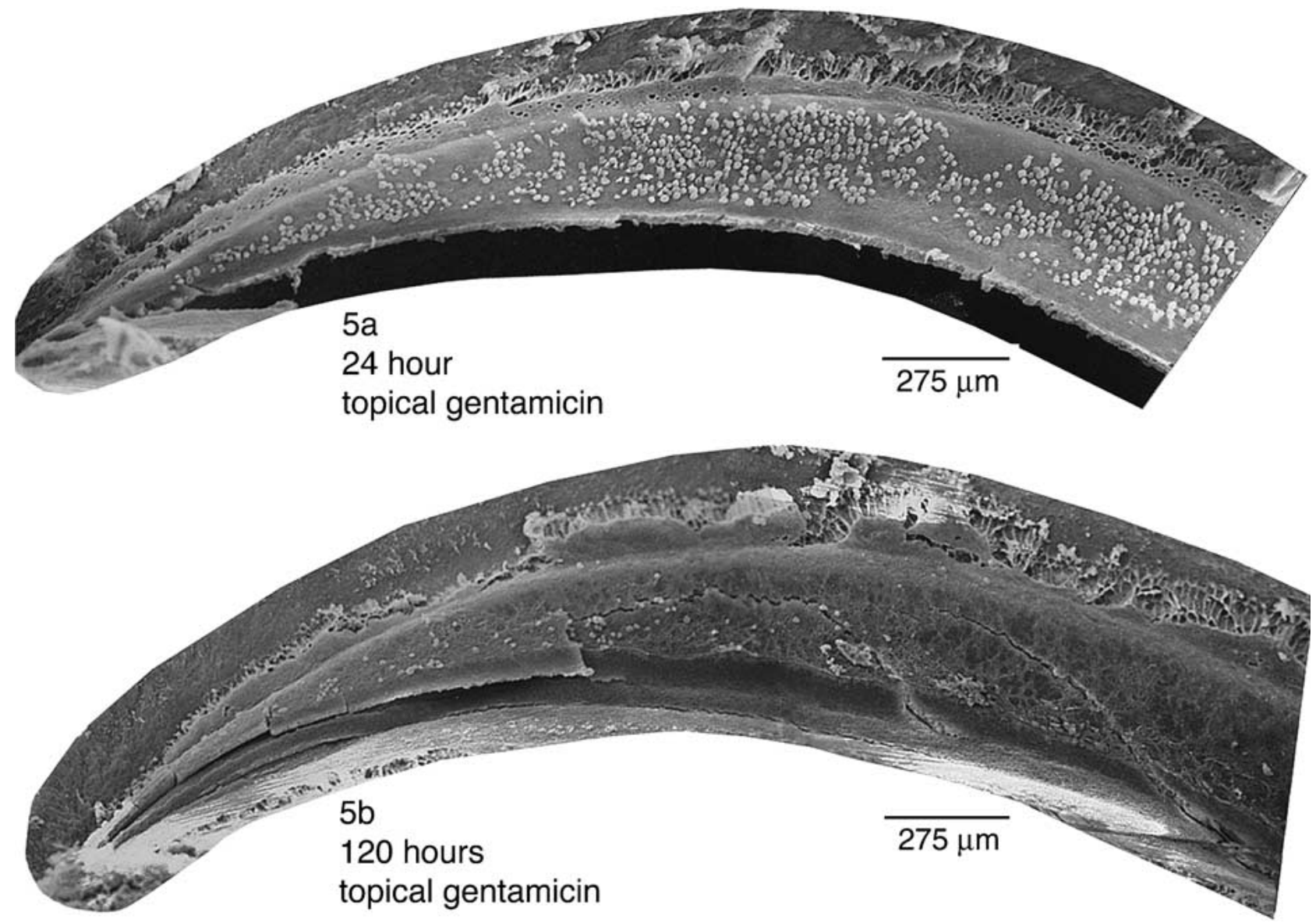

FIG. 5. Montages of SEM images from cochleae (figure shows approximately the basal half of the cochlea) following round window topical application of gentamicin at (a) $24 \mathrm{~h}$ and (b) $120 \mathrm{~h}$ post application. The cochlear hair cells in (a) show significant damage

ular symptoms of Meniere's disease. Because of this renewed clinical interest, the importance of scientifically determining the best ototoxic agent and method of administration is gaining momentum in the literature. Several studies have examined these aspects using mammalian animal models such as the chinchilla (Hoffer et al. 2001; Plontke et al. 2002).

In our lab, we are interested in utilizing a topical administration of gentamicin to provide a unilateral ototoxic lesion in the avian model. We study the effects of cochlear hair cell loss and subsequent regeneration on the second-order neurons (nucleus magnocellularis, NM). Because these neurons receive solely unilateral afferent input, manipulating one cochlea allows us to have a within-animal control. The topical application of gentamicin also allows us to create larger regions of damage, even extending throughout the entire cochlea, without the fatal nephrotoxic effects that occur with a systemic dose high enough to achieve similar cochlear lesions. In the current study, the first comparisons in the avian species are made between the gentamicin levels to through 75 of the cochlea with many mature hair cells being extruded from the sensory epithelium. Most of the mature hair cells in (b) have been damaged and subsequently lost throughout the entire length of the cochlea. A few extruding hair cells are seen.

which the cochlear hair cells are exposed by either systemic injection or round window application of gentamicin.

The results obtained from the systemic injection of high-dose gentamicin $(200 \mathrm{mg} / \mathrm{kg})$ show extremely high levels of the drug $(216 \mu \mathrm{g} / \mathrm{ml})$ in the serum as early as $1 \mathrm{~h}$ post-injection. To provide a reference, the peak recommended serum concentration for human therapeutic use is $4-8 \mu \mathrm{g} / \mathrm{ml}$. Despite the high serum levels noted with the systemic injection, the amount of gentamicin that is measured in the perilymph approaches only $13 \mu \mathrm{g} / \mathrm{ml}$. This concentration of gentamicin in the perilymph produces damage to the hair cells in the basal $40 \%$ of the cochlea. Thus, a very high dose of gentamicin is needed to produce a cochlear lesion. Because of this, it is critical to determine the highest dose that is tolerable to a species, particularly with respect to nephrotoxicity, that will also produce a significant and reliable loss of hair cells for study of the auditory system. Our experience has been that in broiler chickens, $200 \mathrm{mg} / \mathrm{kg}$ of gentamicin is a high dose that is well-tolerated. A study of injecting 
$100 \mathrm{mg} / \mathrm{kg}$ of gentamicin into rats showed similar timing results (Tran Ba Huy et al. 1988). Plasma concentrations peaked after $30 \mathrm{~min}$ and organ of Corti concentrations peaked after $3 \mathrm{~h}$. A study of injecting $100 \mathrm{mg} / \mathrm{kg}$ of gentamicin into guinea pigs also found similar timing results with peak serum at $1 \mathrm{~h}$ and peak perilymph at $2 \mathrm{~h}$ (Federspil et al. 1976).

In our systemic injection group, both cochleae were equally exposed to gentamicin and a regression analysis shows a good correlation between the right and left ear gentamicin concentrations measured in the perilymph. This supports our experience in the impressive lack of variability between right and left cochleae in terms of hair cell loss and regeneration following systemic injections of gentamicin. Not only is the percent length damage always identical, but the pattern of damage at the transition zone between damaged and normal hair cells is also identical between cochleae of the same chicken.

The round window application of gentamicin produced significantly more damage to the cochlear hair cells than obtainable with the systemic injections. These anatomic observations via SEM are consistent with measurements of gentamicin in the perilymph of $55 \mu \mathrm{g} / \mathrm{ml}$ detected at $4 \mathrm{~h}$ after topical application. These perilymph gentamicin levels are 4 times the concentration that can be obtained following the traditional systemic injection. Interestingly, the amounts of gentamicin in our study are considerably lower than those reported in a chinchilla model $4 \mathrm{~h}$ after topical application of gentamicin by a factor of about 3 (Hoffer et al. 2001). However, the administration methods were different. We used a pledget for $2 \mathrm{~h}$, while they used a microcatheter attached to an osmotic pump for 7 days. Their gentamicin peak was seen $72 \mathrm{~h}$ after drug ( 7 days of administration) while ours was $4 \mathrm{~h}$ after drug ( $2 \mathrm{~h}$ of administration). Hoffer et al. (2001) also reported amounts of gentamicin in the chinchilla perilymph following the administration of a single dose of gentamicin using a microcatheter without an attached pump. The microcatheter was inserted into the round window niche and the tube was filled with approximately $200 \mu \mathrm{l}$ of gentamicin $(5 \mathrm{mg} / \mathrm{ml})$. The drug diffused down the catheter and across the round window membrane via gravity and osmosis (method information via personal communication with Dr. Hoffer). Their results for this method were similar in timing to ours (peak at $4 \mathrm{~h}$, cleared by $72 \mathrm{~h}$ ). However, the peak amount is $700 \mu \mathrm{g} / \mathrm{ml}$, which is nearly 10 times the amount we measured and 2 times the peak amount they measured when using the osmotic pump. Clearly, the species used can result in large differences. Also, differences in method of administration amount to very large differences in the amount of gentamicin in the perilymph, as shown by
Hoffer et al. (2001). We agree with Hoffer and colleagues who note that generalizing these results to humans must be approached with great caution.

The anatomical damage detected in our study from the round window technique shows near-complete cochlear hair cell ablation with much of the damage arising in the first $24 \mathrm{~h}$. This damage occurs earlier and is much more extensive than that found in chickens treated with a systemic injection. Gentamicin is also cleared from the perilymph much more slowly after topical application with significant reductions not seen until after $24 \mathrm{~h}$ versus $12 \mathrm{~h}$ after the systemic administration. However, gentamicin levels remained statistically significantly higher in the inner ear in both systemic and topical administrations at $120 \mathrm{~h}$ when compared with controls. Thus, complete clearing of the drug takes longer than 5 days for either administration technique.

With the round window technique, gentamicin was somewhat surprisingly found in the serum and contralateral cochlea at low levels. However, there was no evidence of anatomical or structural damage in the contralateral cochlea by SEM. It is possible that any effect is too slight to be seen at the low-power SEM that we utilized. Possible effects could also be seen at the ultrastructural level or noted physiologically. We did not use these other measures and recognize the need to examine them in future experiments to ensure that the gentamicin is truly not affecting the contralateral cochlea.

The pathway of absorption into the serum and contralateral perilymph can be partially explained by the mucosal contact of gentamicin in the middle ear at the time of gentamicin pledget application. However, the contralateral cochlea has gentamicin levels that exceed serum levels, suggesting that more than just systemic absorption is responsible. The anatomy of the chicken middle ear is noteworthy as it has a direct tubal pathway connecting the right and left middle ear tympanic cavities. With this technique it is possible that a small quantity of gentamicin could be transferred to the contralateral middle ear and detected by our sampling. Subsequently, this might be picked up when inserting the needle through the round window to harvest perilymph, or it might be absorbed into the perilymph through the round window membrane prior to harvest. Our experience in developing this topical administration method showed us that dripping gentamicin directly on the round window was not an effective method of damaging cochlear hair cells (Husmann et al. 1998) and thus we favor the former possibility for explaining gentamicin levels in the contralateral perilymph.

Gentamicin was also surprisingly found in the serum and perilymph of control chickens treated with topical saline and systemic saline. No anatomical 
damage was detected in the scanning electron micrographs of these chickens' cochleae. The gentamicin levels detected were extremely low and thus may display the sensitivity limits of the gentamicin assay used. Since all of the gentamicin levels were collected using the same assay, they would all be slightly increased by the same amount. Thus, statistical analyses comparing the groups would not be affected. Statistical analyses showed no differences in the amounts of gentamicin found when comparing the systemic and topical methods, so the trace amounts detected are not related to the method of drug administration.

The variability in gentamicin levels that can be expected between different chickens following systemic injection was compared with the variability observed between chickens following round window application of gentamicin. The round window technique had significantly more variance between birds than noted with systemic injection. This variability was seen anatomically by Husmann et al. (1998) with a wide range of cochlear hair cell damage being observed, especially at lower gentamicin doses than used in our current study. In the 1998 study, this was attributed to the subtle differences in the position of the gentamicin pledget at the round window or to possibly different levels of sensory epithelium biologic susceptibility that may differ among birds. Our current study would support the former possibility, given the higher variability in perilymph levels with the topical delivery compared with the systemic delivery. The placement of the gentamicin pledget at the round window resulting in different rates of diffusion seems to be the most likely explanation for the variability between these two techniques.

\section{CONCLUSIONS}

The systemic injection of gentamicin appears to be the most reliable technique for avoiding any significant interbird variability. The cochlear damage can be well correlated between the two ears of the same bird. The shortcoming of the systemic injection is the ability to damage only a limited portion of the cochlea secondary to increased serum levels and lethal nephrotoxicity.

The ability to create significantly more cochlear hair cell damage is noted with the round window application of gentamicin. This allows study of loss and regeneration of apical hair cells, responsible for transduction of low-frequency sound, which usually are not damaged following systemic gentamicin administration. This topical technique, although appearing to have more interbird variability than the traditional systemic injections, can also be utilized when it is necessary to have an intrabird anatomical control for comparison (i.e., contralateral, untreated cochlea). The round window technique also spares significant exposure to the remainder of the animal which may decrease any possibility of confounding results.

These two methods of gentamicin administration offer different advantages and disadvantages to the researcher. Researchers will need to weigh these pros and cons and then determine which method is superior for each individual experiment.

\section{ACKNOWLEDGMENTS}

We would like to thank Sandy Parsons for excellent histological assistance and the University of Kansas Medical Center Clinical Laboratory Department for conducting the gentamicin assays. This work was supported by NIDCD R01 DC01589 to DD and by the University of Kansas Medical Center Department of Otolaryngology Head and Neck Surgery.

\section{REFERENCES}

Cotanche DA, Lee KH, Stone JS, Picard DA. Hair cell regeneration in the bird cochlea following noise damage or ototoxic drug damage. Anat. Embryol. 189:1-18, 1994.

Federspil P, Schatzle W, Tiesler E. Pharmacokinetics and ototoxicity of gentamicin, tobramycin, and amikacin. J. Infect. Dis. 134(Suppl):S200-S205, 1976.

Girod DA, Duckert LG, Rubel EW. Possible precursors of regenerated hair cells in the avian cochlea following acoustic trauma. Hear. Res. 42:175-194, 1989.

Girod DA, Tucci DL, Rubel EW. Anatomical correlates of functional recovery in the avian inner ear following aminoglycoside ototoxicity. Laryngoscope 101:1139-1149, 1991.

Hoffer ME, Allen K, Kopke RD, Weisskopf P, Gottshall K, Wester D. Transtympanic versus sustained-release administration of gentamicin: kinetics, morphology, and function. Laryngoscope 111:1343-1357, 2001.

Husmann KR, Morgan AS, Girod DA, Durham D. Round window administration of gentamicin: A new method for the study of ototoxicity of cochlear hair cells. Hear. Res. 125:109-119, 1998.

LiPPE WR, WEstbrook EW, RyALs BM. Hair cell regeneration in the chicken cochlea following aminoglycoside toxicity. Hear. Res. 56:203-210, 1991.

Marean GC, Burt JM, Beecher MD, Rubel EW. Hair cell regeneration in the European starling (Sturnus vulgaris): Recovery of pure-tone detection thresholds. Hear. Res. 71:125-136, 1993.

Plontke SK, Wood AW, Salt AN. Analysis of gentamicin kinetics in fluids of the inner ear with round window administration. Otol. Neurotol. 23:967-974, 2002.

Smolders JW. Functional recovery in the avian ear after hair cell regeneration. Audiol. Neurootol. 4:286-302, 1999.

Tran Ba Huy P, Deffrennes D. Aminoglycoside ototoxicity: influence of dosage regimen on drug uptake and correlation between membrane binding and some clinical features. Acta Otolaryngol. 105:511-515, 1988.

Tsue TT, Oesterle EC, Rubel EW. Hair cell regeneration in the inner ear. Otolarynlol. Head Neck Surg. 111:281-301, 1994.

Tucci DL, Rubel EW. Physiologic status of regenerated hair cells in the avian inner ear following aminoglycoside ototoxicity. Otolaryngol. Head Neck Surg. 103:443-450, 1990. 